\title{
Impact of Stent Platform of Paclitaxel-Eluting Stents
}

\section{- Assessment of Neointimal Distribution on Optical Coherence Tomography -}

Hiromasa Otake, MD; Junya Shite, MD; Toshiro Shinke, MD; Naoki Miyoshi, MD; Amane Kozuki, MD; Hiroyuki Kawamori, MD; Masayuki Nakagawa, MD; Ryoji Nagoshi, MD; Hirotoshi Hariki, MD; Takumi Inoue, MD; Tsuyoshi Osue, MD; Yu Taniguchi, MD; Noritoshi Hiranuma, MD; Ryo Nishio, MD; Hiroto Kinutani, MD; Ken-ichi Hirata, MD

\begin{abstract}
Background: The Taxus Express ${ }^{\mathrm{TM}}$ paclitaxel-eluting stent (Express-PES) and Taxus LibertéTM PES (Liberté-PES) have identical drugs, drug doses, and polymers, but different stent platforms. The Liberté-PES platform has thinner struts, specifically designed for more uniform drug elution.
\end{abstract}

Methods and Results: Fifty-four patients who underwent 6-month follow-up optical coherence tomography (OCT) after Express-PES $(n=27)$ or Liberté-PES $(n=27)$ implantation were enrolled. Longitudinal and circumferential uniformity of neointimal distribution was evaluated in 3-D by computing mean neointimal thickness (NIT) within 360 equally spaced radial sectors for every 1-mm cross-section. After stenting, intravascular ultrasound showed that Liberté-PES had a significantly smaller maximum angle between adjacent struts, with a tendency toward a lower incidence of \% length of the segment with maximum angle $>90^{\circ}$ than Express-PES. Liberté-PES had a significantly thinner mean NIT than the Express-PES with comparable frequency of uncovered struts. Longitudinal and circumferential absolute variation of NIT expressed by standard deviation of NIT from each sector was significantly smaller for Liberté-PES than for Express-PES. Liberté-PES had a tendency toward a decreased incidence of thrombus and peri-strut low-intensity areas (findings suggestive of delayed arterial healing), compared to Express-PES.

Conclusions: Stent design and thickness appeared to affect neointima suppression of PES. The stent platform of the Liberté-PES may offer greater and more homogeneous reduction of neointimal proliferation spatially across the full length of the PES. (Circ $J$ 2012; 76: 1880-1888)

Key Words: Optical coherence tomography; Paclitaxel-eluting stents; Stent design; Thin struts

$\mathbf{S}$ ince the concept of stenting was first proposed more than 30 years ago, ${ }^{1}$ a variety of factors, such as stent design, ${ }^{2-4}$ strut thickness, ${ }^{5,6}$ and material, ${ }^{7}$ have been demonstrated to affect neointimal proliferation after bare-metal stent (BMS) implantation. Among these factors, because inhomogeneous strut placement of drug-eluting stents (DES) dramatically affects local drug concentrations, ${ }^{8}$ stent design is one of the most important factors in the safety and efficacy of DES, by influencing uniform vessel healing and homogenous suppression of neointimal proliferation. Also, thinner stent struts reduce the incidence of BMS restenosis, regardless of differences in stent design. ${ }^{6,9}$ No clinical report, however, has clearly demonstrated the relationship between stent design, strut thickness, and detailed arterial response after DES therapy, probably due the lack of established methods to quantitatively evaluate in vivo variability of neointima distribution in a multidimensional fashion.

The Taxus Express ${ }^{\mathrm{TM}}$ paclitaxel-eluting stent (Taxus Express PES; Boston Scientific, Natick, MA, USA) and Taxus LibertéTM PES (Taxus Liberte PES; Boston Scientific) have identical drugs, drug doses, and polymers, but different stent platforms. The Taxus Liberte PES platform has thinner struts $(0.038$ in vs. $0.052 \mathrm{in} ; 0.097 \mathrm{~mm}$ vs. $0.13 \mathrm{~mm}$ ) specifically designed for achieving uniform drug elution (Figure 1). Given that the only differences between the Taxus Express PES and Taxus Liberté PES are the stent design and strut thickness, we hypothesized that we could clarify the impact of these factors on vessel reaction in the early phase after DES therapy by comparing 6-

Received November 25, 2011; revised manuscript received April 3, 2012; accepted April 8, 2012; released online May 19 , 2012 Time for primary review: 23 days

Division of Cardiovascular and Respiratory Medicine, Department of Internal Medicine, Kobe University Graduate School of Medicine, Kobe, Japan

Mailing address: Junya Shite, MD, Department of Cardiology, Kobe University Graduate School of Medicine, 7-5-1 Kusunoki-cho, Chuoku, Kobe 650-0017, Japan. E-mail: shite@med.kobe-u.ac.jp

ISSN-1346-9843 doi:10.1253/circj.CJ-11-1363

All rights are reserved to the Japanese Circulation Society. For permissions, please e-mail: cj@j-circ.or.jp 


\begin{tabular}{lcc}
\hline & Taxus $^{\circledR}$ Liberté $^{\circledR}$ & Taxus $^{\circledR}$ Express $^{\circledR}$ \\
\hline & & \\
Stent Pattern & $316 \mathrm{~L}$ stainless steel & $316 \mathrm{~L}$ stainless steel \\
Metal strut thickness & $0.0038^{\prime \prime}$ & $0.0052 "$ \\
Polymer & Translute $^{\mathrm{TM}}$ & Translute \\
Drug & Paclitaxel & Paclitaxel \\
Loading dose/stent & $112 \mu \mathrm{g} / 3.0 \times 16 \mathrm{~mm}$ & $108 \mu \mathrm{gg} / 3.0 \times 16 \mathrm{~mm}$ \\
Release Kinetics & $10 \%$ & $10 \%$ \\
(\% released in 28 days $)$ & $(10 \%: 30$ days $)$ & $(10 \%: 30$ days $)$ \\
Drug: polymer ratio & $1: 9$ & $1: 9$ \\
\hline
\end{tabular}

Figure 1. Comparison of device description. The only differences between the Taxus Express paclitaxeleluting stent (PES) and Taxus Liberte PES were in the strut thickness and stent design.
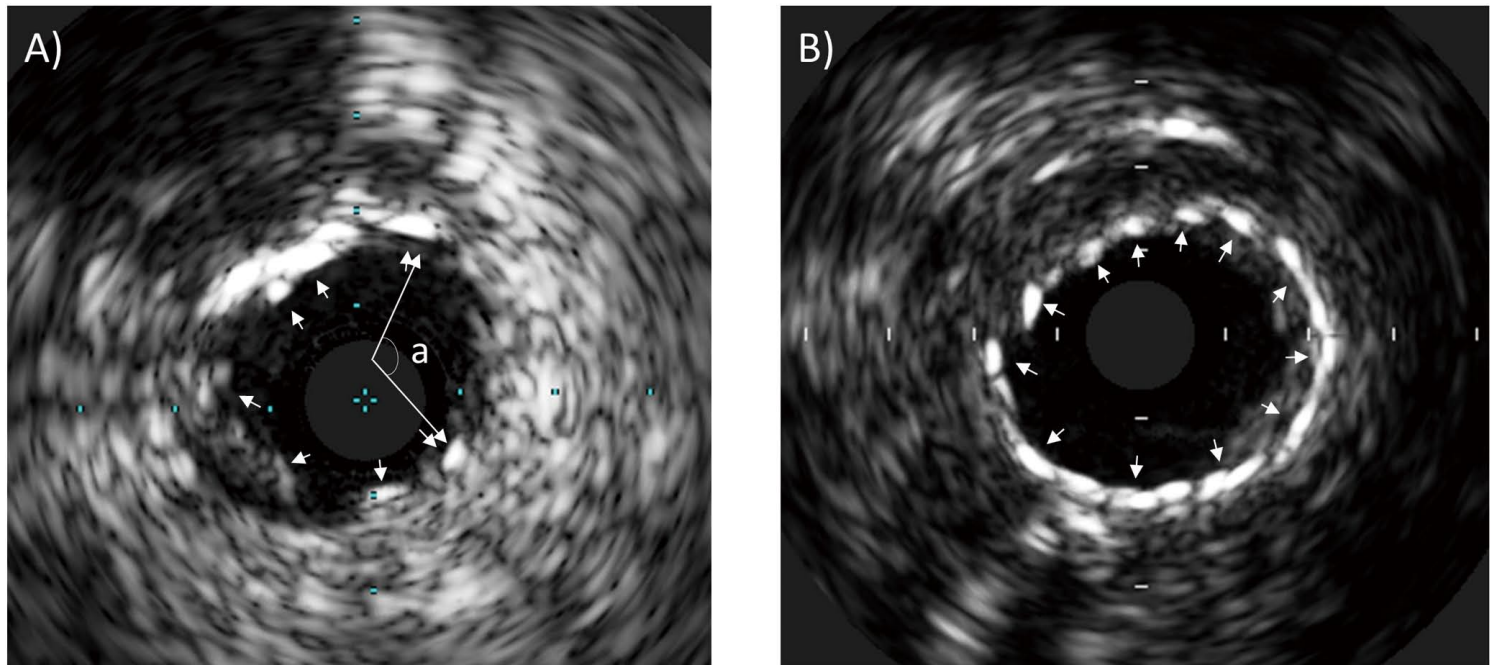

Figure 2. Stent strut distribution on intravascular ultrasound after stenting: the maximum angle (a) between adjacent struts was defined as interstrut angle. (A) Representative image of non-uniform strut distribution of the Taxus Express paclitaxel-eluting stent (PES). (B) Representative image of uniform strut distribution of the Taxus Liberte PES

month follow-up optical coherence tomography (OCT) findings of these PES. The aim of this study was to investigate the role of stent design and strut thickness on neointima suppression in the early phase after PES implantation using the newly developed OCT-based 3-D assessment of neointimal distribution.

\section{Methods}

\section{Subjects}

In this retrospective observational study, consecutive patients treated with Taxus Express ${ }^{\mathrm{TM}}$ PES (Boston Scientific) and Taxus Liberté ${ }^{T M}$ PES (Boston Scientific) between May 2007 and July 2009 who fulfilled the inclusion and exclusion crite- ria were selected. The inclusion criteria for the study were: (1) de novo native coronary lesion ( $>50 \%$ diameter stenosis) treated with Taxus Express or Taxus Liberté PES, followed up on routine 6-month coronary angiography with follow-up OCT; (2) native vessel size $2.5-3.5 \mathrm{~mm}$ in diameter; and (3) stable or unstable angina pectoris. The exclusion criteria included: (1) ST-elevated acute myocardial infarction; (2) contraindication for dual antiplatelet therapy; (3) left main coronary artery disease; and (4) congestive heart failure. During this period, OCT was performed if the following criteria were met: (1) the target artery was anatomically suitable for OCT according to previously described criteria; ${ }^{10}$ and (2) written informed consent for OCT was obtained from the patient. Finally, we enrolled a total of 54 consecutive patients treated with the Taxus 
A)

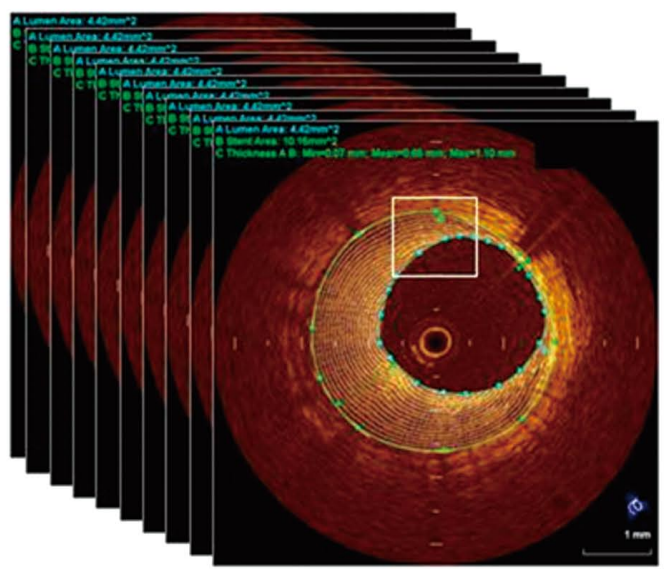

C)

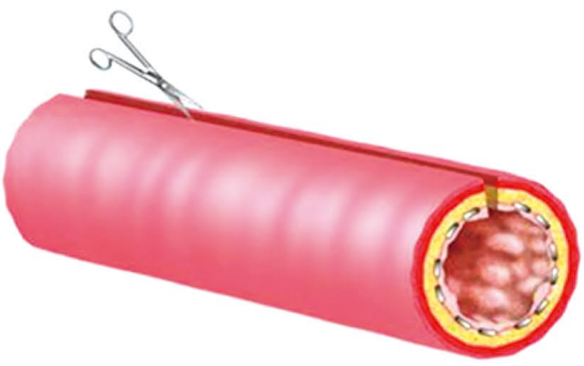

B)
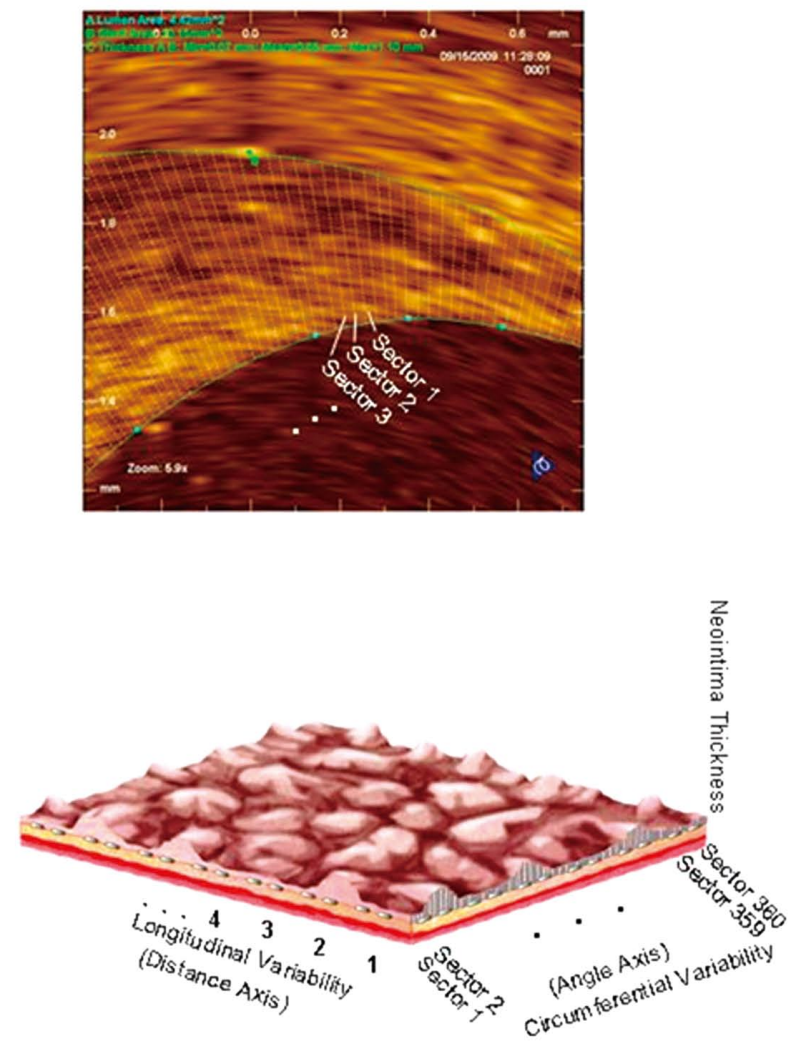

Figure 3. 3-D assessment of neointimal distribution. (A) Lumen and stent area are manually traced every $1 \mathrm{~mm}$. (B) Neointimal area is divided into 360 circumferential sectors ( $1^{\circ}$ for each sector as referenced from the lumen center). Mean neointimal thickness (NIT) is then obtained for each sector, and the computation is performed for the entire stented segment at 1-mm intervals. (C) To visualize the 2-D image layout, the stent surface is cut along the longitudinal direction and then flattened out into a rectangular shape. The angle (sector) axis represents circumferential variability, and the distance axis indicates longitudinal variability of NIT. For assessing the longitudinal variability of NIT, NIT within the same sector was corrected from different cross-sections. Longitudinal SD was then calculated for each sector. Finally, the longitudinal SD obtained for each sector was averaged across the 360 different sectors within the same stent and defined as the longitudinal variability score of the stent. For the assessment of circumferential variability of NIT, the SD of NIT within the same cross-section was calculated for each cross-section, and then averaged along the entire stented segment (circumferential variability score).

Express PES (27 patients, 27 lesions) or the Taxus Liberté PES (27 patients, 27 lesions). Percutaneous coronary intervention (PCI) was performed under intravascular ultrasound (IVUS) guidance (Boston Scientific or Volcano, Rancho Cordova, CA, USA). All patients were taking aspirin ( $100 \mathrm{mg} /$ day $)$. After stenting, patients were prescribed ticlopidine $(200 \mathrm{mg} /$ day $)$ or clopidogrel ( $75 \mathrm{mg} /$ day). The study was approved by the ethics committee of Kobe University and written informed consent for the follow-up OCT and for inclusion in this study was obtained from each patient.

\section{Quantitative Angiography}

Coronary angiograms, obtained at baseline and at the 6-month follow-up, were analyzed using a computer-based system with edge-detection technique (Medis Medical Imaging Systems, Leiden, Netherlands) by investigators blinded to the clinical presentation, lesion characteristics, and stent assignment. Binary restenosis was defined as $\geq 50 \%$ diameter stenosis of the treated lesion at angiographic follow-up.

\section{Pre- and Post-Intervention IVUS}

Pre-intervention IVUS was interpreted by an experienced observer, who was blinded to clinical and angiographic information. A plaque with predominantly $(>75 \%)$ low echogenic appearance (compared with adventitia) was classified as soft. Conversely, a plaque was considered fibrotic if it was found to be hyperechoic (compared with adventitia). Calcified plaque was classified as such in the presence of acoustic shadowing. ${ }^{11}$

Post-stenting IVUS was also evaluated for the assessment of uniform strut distribution of the deployed stent. The maximum angle between adjacent struts with a protractor centered on the stent was defined as interstrut angle (Figure 2). ${ }^{12}$ Nonuniform strut distribution (NSD) was then defined as interstrut angle $>90^{\circ}$, and stents were defined as having NSD if, for any segment within a stent, NSD could be continuously observed for $>0.5 \mathrm{~mm}$ longitudinally, as defined in a previous IVUS report. Percent NSD was defined as the length of the segment with NSD divided by stent length. ${ }^{13}$ 


\begin{tabular}{|c|c|c|c|}
\hline & $\begin{array}{l}\text { Taxus Liberté } \\
\text { PES }(n=27)\end{array}$ & $\begin{array}{l}\text { Taxus Express } \\
\text { PES }(\mathrm{n}=27)\end{array}$ & $P$ value \\
\hline \multicolumn{4}{|l|}{ Patient characteristics } \\
\hline Age (years) & $69.1 \pm 9.9$ & $69.9 \pm 10.9$ & 0.78 \\
\hline Male gender & $18(33)$ & $26(96)$ & $<0.0001$ \\
\hline Hypertension & $21(78)$ & $20(74)$ & 0.75 \\
\hline Diabetes & $9(33)$ & $14(52)$ & 0.17 \\
\hline Smoking & $15(56)$ & $9(33)$ & 0.10 \\
\hline Hyperlipidemia & $20(74)$ & $16(59)$ & 0.25 \\
\hline Angina status & & & $>0.99$ \\
\hline Stable & $22(19)$ & $22(19)$ & \\
\hline Unstable & $5(81)$ & $5(81)$ & \\
\hline Follow-up days & $214 \pm 30$ & $211 \pm 69$ & 0.84 \\
\hline Dual antiplatelet therapy at 6 months & $22(82)$ & $26(96)$ & 0.19 \\
\hline Vessel treated & & & 0.76 \\
\hline LAD/RCA/LCX (\%) & $44 / 19 / 37$ & $44 / 26 / 30$ & \\
\hline Lesion location & & & 0.38 \\
\hline Proximal & $7(26)$ & $10(37)$ & \\
\hline Mid/Distal & $20(74)$ & $17(63)$ & \\
\hline AHA/ACC lesion classification & & & 0.77 \\
\hline A/B1/B2/C (\%) & 22/40/19/19 & $15 / 37 / 29 / 19$ & \\
\hline \multicolumn{4}{|l|}{ Procedural characteristics } \\
\hline Stent length (mm) & $20.5 \pm 6.9$ & $20.3 \pm 6.8$ & 0.91 \\
\hline Stent diameter $(\mathrm{mm})$ & $2.91 \pm 0.37$ & $3.02 \pm 0.35$ & 0.26 \\
\hline Maximum inflation pressure (atm) & $14.6 \pm 3.1$ & $15.0 \pm 3.2$ & 0.69 \\
\hline \multicolumn{4}{|c|}{ Quantitative coronary angiography before $\mathrm{PCl}$} \\
\hline Lesion length $(\mathrm{mm})$ & $12.8 \pm 7.5$ & $14.1 \pm 5.0$ & 0.48 \\
\hline Reference diameter (mm) & $2.50 \pm 0.58$ & $2.53 \pm 0.50$ & 0.85 \\
\hline Minimum lumen diameter $(\mathrm{mm})$ & $0.38 \pm 0.21$ & $0.34 \pm 0.22$ & 0.46 \\
\hline \% Diameter stenosis (\%) & $84.2 \pm 7.9$ & $86.2 \pm 8.6$ & 0.37 \\
\hline
\end{tabular}

Data given as mean $\pm \mathrm{SD}$ or $\mathrm{n}(\%)$.

PES, paclitaxel-eluting stent; LAD, left anterior descending artery; RCA, right coronary artery; LCX, left circumflex artery;

AHA, American Heart Association; ACC, American College of Cardiology; PCl, percutaneous coronary intervention.

\section{OCT}

OCT was performed as previously reported. ${ }^{14}$ Briefly, a $0.016-$ in OCT catheter (ImageWire, LightLab Imaging, Westford, MA, USA) was advanced to the distal end of the stented lesion through an occlusion balloon catheter (Helios ${ }^{\mathrm{TM}}$; LightLab Imaging). The occlusion balloon was inflated to $0.5 \mathrm{~atm}$ at the proximal site of the stented lesion, followed by an infusion of lactated Ringer's solution into the coronary artery from the distal tip of the occlusion balloon catheter at $0.5 \mathrm{ml} / \mathrm{s}$, serving as a flush to clear the area of blood. The entire stented length was then imaged using an automatic pullback system moving at $1 \mathrm{~mm} / \mathrm{s}$.

Image Analysis All images were analyzed by independent observers who were masked to the clinical presentation, lesion characteristics, and stent assignment. For the quantitative analysis, cross-sectional OCT images were analyzed at 1-mm intervals. Bifurcation cross-sections with side branches were excluded from this analysis. Neointimal thickness (NIT) inside each stent strut and stent area was measured. Stent and lumen area were manually measured and neointima area was calculated as stent area-lumen area. Struts with measured $\mathrm{NIT}=0 \mu \mathrm{m}$ were defined as uncovered struts. The frequency of uncovered struts was calculated as the number of uncovered struts divided by the total number of struts for each stent. To assess for stent asymmetric expansion, a stent eccentricity index (SEI) was determined by the minimum stent diameter divided by the maximum stent diameter in each cross-section. Then, average and minimum SEI were calculated for each stent. ${ }^{15}$ Intracoronary thrombus was defined as a protruding mass beyond the stent strut into the lumen with significant attenuation behind the mass. ${ }^{16,17}$ To differentiate thrombus from plaque protrusion or neointimal hyperplasia, we excluded protruding masses without significant signal attenuation and surface irregularity. ${ }^{15}$ According to previous pathological investigations, peri-strut low intensity area (PLIA) observed after DES deployment may represent the presence of fibrin or proteoglycan, probably reflecting the extent of delayed healing or impaired neointimal maturation. ${ }^{18,19}$ Therefore in the present study, we assessed the frequency of stent struts with PLIA, which was defined as a region around the stent struts with a homogenous lower intensity appearance than the surrounding tissue on OCT without significant signal attenuation behind the area.

\section{3-D Assessment of Neointimal Distribution}

Because the platform of the Taxus Liberté PES was engineered specifically to produce consistent drug elution, we evaluated the uniformity of neointima suppression within the stented segment in order to compare the variability of neointima proliferation of Taxus Liberté PES with that of the Taxus Express PES. For this purpose, we conducted a 3-D assessment of instent neointimal distribution. First, lumen and stent contours 


\begin{tabular}{|lccc|}
\hline Table 2. IVUS Measurements & Taxus Liberté & Taxus Express & P value \\
PES (27 lesions) & PES (27 lesions) & \\
Qualitative IVUS findings before stenting & $5(19)$ & $6(22)$ & 0.81 \\
Soft & $8(30)$ & $6(22)$ & \\
Fibrous & $14(51)$ & $15(56)$ & \\
$\quad$ Calcified & & & \\
Quantitative IVUS findings after stenting & $36.7 \pm 25.1$ & $61.3 \pm 32.6$ & 0.003 \\
Maximum interstrut angle $\left({ }^{\circ}\right)$ & $3(11)$ & $8(30)$ & 0.18 \\
Lesions with NSD & $0.5 \pm 1.4$ & $4.0 \pm 8.9$ & 0.07 \\
Percent NSD (\%) & & & \\
\hline
\end{tabular}

Data given as mean \pm SD or $n(\%)$.

IVUS, intravascular ultrasound; PES, paclitaxel-eluting stent; NSD, non-uniform strut distribution.

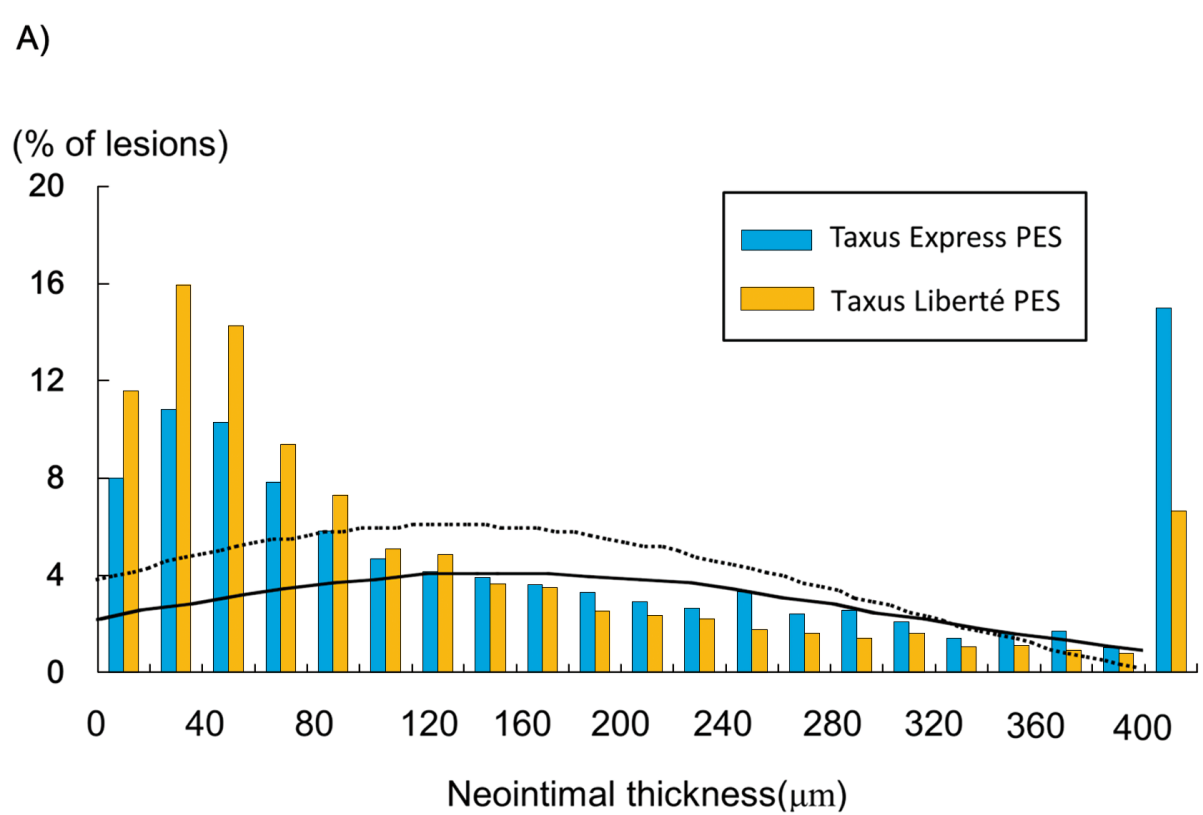

B)

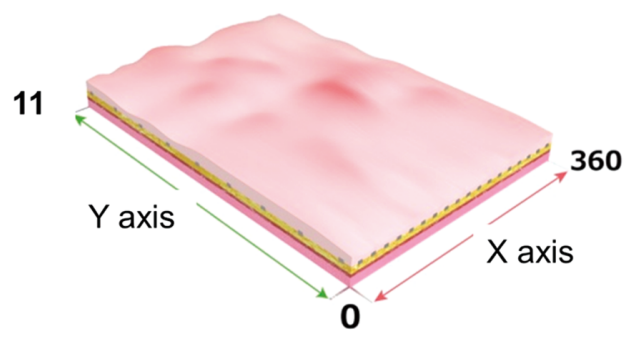

TAXUS Liberté PES

Average NIT: $83 \mu \mathrm{m}$

Circumflex variability score: 0.083

Longitudinal variability score: 0.065

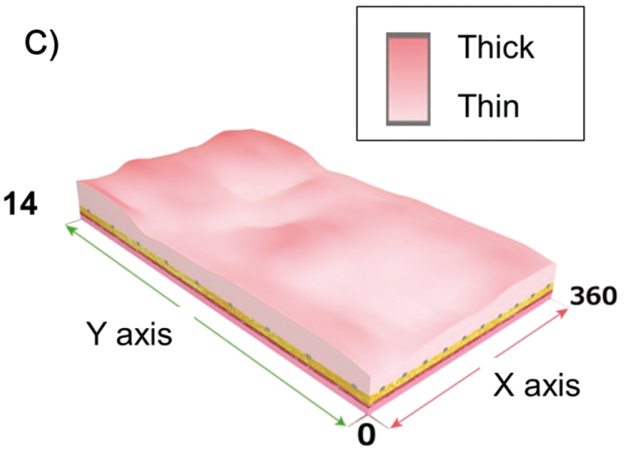

TAXUS Express PES

Average NIT: $160 \mu \mathrm{m}$

Circumflex variability score: 0.16

Longitudinal variability score: 0.12

Figure 4. Distribution of neointimal thickness and representative cases of Taxus Liberté and Taxus Express paclitaxel-eluting stents (PES). (A) Distribution of neointimal thickness. (B) Representative case of Taxus Liberté PES. Average neointimal thickness (NIT), $83 \mu \mathrm{m}$; circumferential variability score, 0.083; longitudinal variability score, 0.065 . (C) Representative case of Taxus Express PES. Average NIT, $160 \mu \mathrm{m}$; circumferential variability score, 0.16 ; longitudinal variability score, 0.12 . 


\begin{tabular}{lccc}
\hline Table 3. OCT Measurements & & & \\
& $\begin{array}{c}\text { Taxus Liberté } \\
\text { PES (27 lesions) }\end{array}$ & $\begin{array}{c}\text { Taxus Express } \\
\text { PES (27 lesions) }\end{array}$ & P value \\
Mean stent area $\left(\mathrm{mm}^{2}\right)$ & $8.0 \pm 2.4$ & $8.2 \pm 2.2$ & 0.67 \\
Mean minimum stent area $\left(\mathrm{mm}^{2}\right)$ & $7.0 \pm 2.4$ & $6.8 \pm 2.2$ & 0.75 \\
Mean SEI & $0.94 \pm 0.02$ & $0.94 \pm 0.02$ & 0.26 \\
Average of minimum SEI & $0.87 \pm 0.04$ & $0.88 \pm 0.04$ & 0.48 \\
Mean lumen area $\left(\mathrm{mm}^{2}\right)$ & $6.4 \pm 2.4$ & $6.2 \pm 2.3$ & 0.73 \\
Mean no. struts & $146 \pm 58$ & $131 \pm 47$ & 0.32 \\
Mean no. uncovered stent struts & $5.5 \pm 7.4$ & $5.3 \pm 8.1$ & 0.93 \\
Frequency of uncovered stent struts $(\%)$ & $4.5 \pm 7.8$ & $4.3 \pm 8.2$ & 0.93 \\
Mean neointimal thickness $(\mu \mathrm{m})$ & $120 \pm 59$ & $198 \pm 129$ & 0.006 \\
Mean neointima area (mm $\left.{ }^{2}\right)$ & $1.6 \pm 0.91$ & $2.1 \pm 1.0$ & 0.06 \\
Median neointimal thickness & $109 \pm 120$ & $174 \pm 120$ & 0.05 \\
Average of max neointimal thickness $(\mu \mathrm{m})$ & $509 \pm 246$ & $716 \pm 253$ & 0.004 \\
Average of maximum minus minimum neointimal thickness $(\mu \mathrm{m})$ & $509 \pm 246$ & $710 \pm 251$ & 0.004 \\
\hline
\end{tabular}

Data given as mean \pm SD or $n(\%)$.

OCT, optical coherence tomography; PES, paclitaxel-eluting stent; SEI, stent eccentricity index.

on cross-sectional images were manually traced at 1 -mm intervals. The dedicated software then portioned the neointima area (between stent and lumen) into 360 equally spaced circumferential sectors (spanning $1^{\circ}$ for each sector) per each cross-sectional OCT image (Figure 3 ). In this process, the lumen center was used as a reference of sector division. Mean NIT in each sector was automatically computed. Subsequently, the variability of NIT within the entire stent was evaluated using 2 newly developed parameters: longitudinal variability score and circumferential variability score. To assess the longitudinal variability of NIT, first, NIT within the same sector was collected from different cross-sections. Then, longitudinal SD was calculated for each sector. Finally, the longitudinal SD obtained from each sector was averaged across the 360 different sectors within the same stent and defined as the longitudinal variability score of the stent. For the assessment of circumferential variability of NIT, the SD of NIT within the same cross-section was calculated for each cross-section, and then averaged along the entire stented segment (circumferential variability score). The longitudinal and circumferential variability score were compared between the Taxus Liberté PES and the Taxus Express PES.

\section{Clinical Follow-up}

The incidence of non-fatal myocardial infarction, stent thrombosis, and target lesion revascularization was evaluated 6 months after the index stent procedure. Target lesion revascularization was defined as any reintervention (surgical or percutaneous) to treat restenosis of the analyzed segment.

\section{Statistical Analysis}

Statistical analysis was performed using StatView 5.0 (SAS Institute, Cary, NC, USA) and SPSS version 16 (SPSS, Chicago, IL, USA). Qualitative data are presented as frequencies and quantitative data are given as mean $\pm \mathrm{SD}$. Categorical variables were compared using chi-squared or Fisher exact test. For continuous variables, comparisons between the Taxus Express and Taxus Liberté PES were performed using a 2-tailed, unpaired t-test. The Welch t-test was used for comparisons of variables with unequal variances (ie, NIT). To assess whether quantification of the frequency of stent struts with PLIA varied with time and between observers, Bland-Altman repeatability analysis was used. ${ }^{20} \mathrm{~A} 2$-tailed $\mathrm{P}<0.05$ was considered statistically significant.

\section{Results}

Baseline patient and lesion characteristics are given in Table $\mathbf{1 .}$ Aspirin was continued throughout follow-up for all patients with DES. Because the subjects with DES were non-randomly selected, there was a higher percentage of male subjects in the Taxus Express PES group. Otherwise, there were no significant differences between the groups. Pre- and post-PCI IVUS measurement are listed in Table 2. Baseline plaque characteristics did not differ between the 2 groups. Final PCI IVUS, however, showed a significantly smaller interstrut angle and a lower number of stents with NSD and \%NSD segment in the Taxus Liberté PES group than in the Taxus Express PES group (Table 2).

Six-month follow-up OCT showed that mean and median NIT was significantly smaller in the Taxus Liberté PES group than in the Taxus Express PES group, despite comparable average stent area, average SEI, and minimum SEI between the 2 groups. NIT distribution is shown in Figure 4A. The frequency of uncovered struts, however, was similar between the 2 groups (Table 3). Regarding neointimal distribution, Taxus Liberte PES had a significantly smaller average maximum NIT and average (max-min) NIT than Taxus Express PES (Table 3). In addition, both circumferential and longitudinal variability of NIT was significantly smaller in the Taxus Liberté PES group than in the Taxus Express PES group (Figure 5A). Representative cases of the 2 stents are shown in Figures $4 \mathrm{~B}, \mathrm{C}$.

The frequency of thrombus attachment tended to be lower in the Taxus Liberté PES group than in the Taxus Express PES group ( $18.5 \%$ vs. $37.0 \%, \mathrm{P}=0.13)$. The incidence of struts with PLIA also tended to be lower in the Taxus Liberté PES group than in the Taxus Express PES group (Figures 5B,C). BlandAltman analysis indicated close agreement of the frequency of PLIA quantification during the measured time interval, with a bias of $-0.2 \%$ and limit of agreement (2SD of the mean difference) of $9.0 \%$ ( $-9.2 \%$ to $8.8 \%)$. As for the interobserver variability, bias was $-0.3 \%$ and limit of agreement was $10.7 \%$ $(-11.0 \%$ to $10.4 \%)$ on Bland-Altman analysis.

At 6-month follow-up angiography, 3 lesions (11.1\%) in the Taxus Liberté PES group and 5 lesions (18.5\%) in the Taxus 


\section{A) Longitudinal Variability Score}

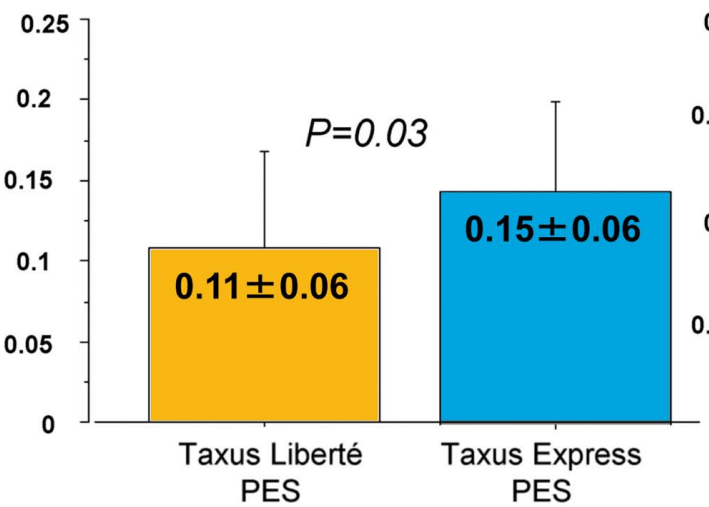

B)

\%)

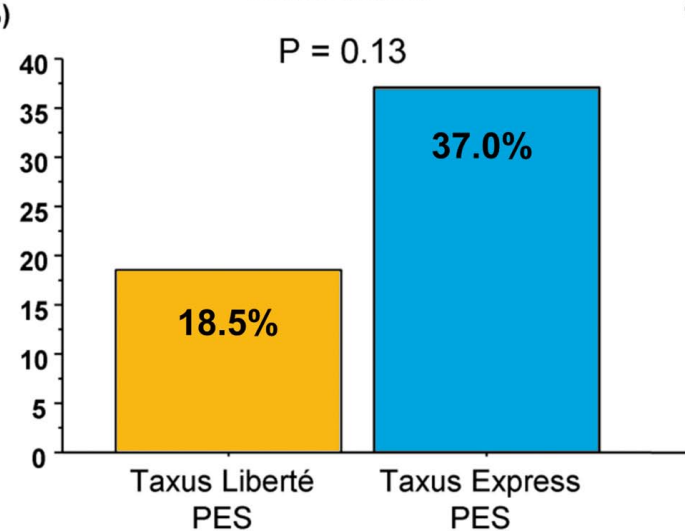

\section{Circumferential Variability Score}

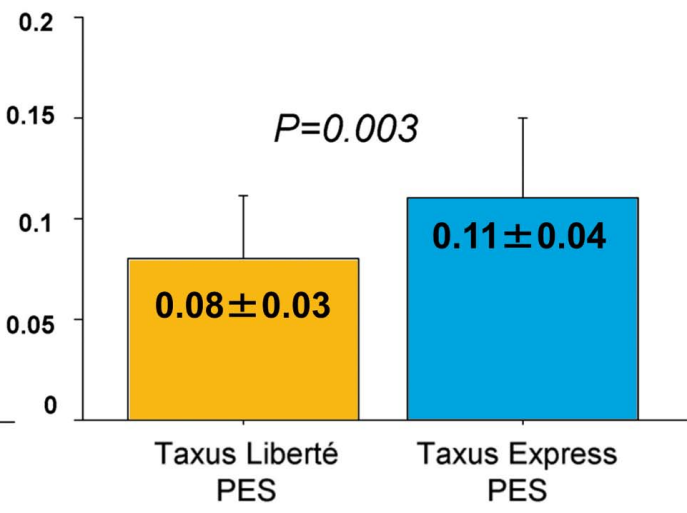

C) Frequency of struts with PLIA

\%)

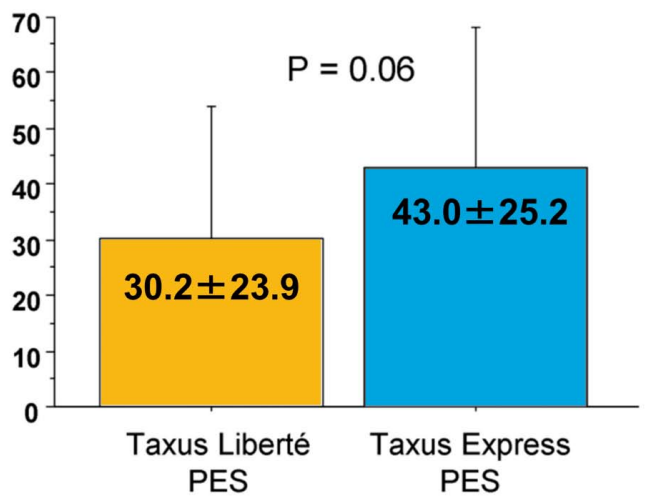

Figure 5. Neointimal variability and the frequency of thrombus and peri-strut low intensity area. (A) Both longitudinal and circumferential variability of neointima thickness were significantly lower in the Taxus Liberté paclitaxel-eluting stent (PES) group than in the Taxus Express PES group. (B) The frequency of thrombus attachment was lower in the Taxus Liberté PES group than in the Taxus Express PES group, although these differences were not statistically significant. (C) The incidence of struts with peri-strut low intensity area (PLIA) tended to be lower in the Taxus Liberté PES group than in the Taxus Express PES group.

Express PES group had binary restenosis $(\mathrm{P}=0.70)$. Target lesion revascularization was required for 2 lesions $(7.4 \%)$ in the Taxus Liberté PES group and 5 lesions (18.5\%) in the Taxus Express PES group $(\mathrm{P}=0.42)$. No stent thrombosis or non-fatal myocardial infarction was observed in these patients up to the 6-month follow-up.

\section{Discussion}

Stent design and strut thickness are thought to impact on the safety and efficacy of DES by affecting the uniformity of vessel healing and homogeneity of neointima suppression. An imaging method, however, has not previously been established to quantitatively evaluate in vivo variability of neointima distribution in a multi-dimensional fashion within an implanted stent. Therefore, the impact of these factors on vessel healing has not been fully evaluated in humans. Although IVUS is a robust tool for the quantification of neointimal tissue, the resolution of IVUS (ie, 100-150 $\mu \mathrm{m}$ ) may not be sufficient for detecting a suppressed, small degree of neointimal hyperplasia after DES implantation. ${ }^{14,21}$ OCT has a higher resolution (10$15 \mu \mathrm{m}$ ) and can clearly visualize thin neointima and other subtle changes in vascular response, ${ }^{22}$ therefore, we developed an OCT-based 3-D method to quantitatively evaluate the variability of neointimal distribution after stenting (ie, the circumferential and longitudinal variability scores).

The Taxus LibertéTM PES is novel in that it is engineered specifically to produce consistent drug elution after stent deployment. Based on a previous experimental study, this unique design delivers paclitaxel more evenly than the Taxus Express PES. ${ }^{23}$ The use of thinner stent struts is also advantageous for decreasing the amount of local injury, ${ }^{24}$ providing greater flexibility, and for improved deliverability. Despite these potential advantages, the impact of these modifications on neointima growth has not been sufficiently evaluated in humans. In the present study, the Taxus Liberté PES provided greater suppression of neointima than the Taxus Express PES, although both stents elute the same drug with almost the same total dose and release kinetics. Of note, although the mean NIT with the Taxus Liberté PES was significantly thinner than that of the 
Taxus Express PES, the frequency of uncovered struts was comparable between the 2 stents. Given that uncovered struts increase the possible risk of future thrombotic events, ${ }^{24}$ the present results suggest that the modification of the stent platform of the Taxus Liberté PES successfully achieved significant reduction of neointima proliferation without sacrificing safety in patients treated with PES. Although detailed mechanisms are still unclear, we speculate that this might stem from the homogenous drug elution produced by the newly designed platform of the Taxus Liberté PES. Indeed, detailed post-PCI IVUS showed that Taxus Liberté PES had a significantly smaller maximum interstrut angle with a tendency toward a lower incidence of stents with NSD and \%NSD segment as compared with Taxus Express PES, suggesting more uniform strut distribution within a Taxus Liberté PES-treated vessel. Also, using the newly developed 3-D assessment of neointimal distribution, we clearly demonstrated that the Taxus Liberté PES had significantly lower circumferential as well as longitudinal variability of NIT, indicating more homogeneous neointimal suppression with the Taxus Liberté PES than with the Taxus Express PES. According to previous experimental and human studies, local drug concentrations and concentration gradients are clearly linked to the biological effect of the drug. ${ }^{8}$ Consistent with this notion, our group has also demonstrated that uneven drug distribution due to non-uniform stent expansion increases the incidence of uncovered struts and unevenness of NIT when using sirolimus-eluting stents. ${ }^{15}$

In addition to the newly designed stent platform, the use of thinner struts may provide another possible explanation for the greater and more uniform distribution of neointima seen with the Taxus Liberté PES. Previous investigators have demonstrated that the use of thin struts dramatically reduces the incidence of in-stent restenosis and target-lesion revascularization after BMS implantation, probably due to decreased local vessel injury during the stenting procedure..$^{3,8}$ The new platform may have a potential benefit of better and more rapid vessel healing. According to a previous pathological report, the presence of PLIA observed on follow-up OCT might represent the presence of fibrin deposition or proteoglycan, both of which can be a marker of delayed vessel healing. ${ }^{18}$ In the current study, the lesions treated with the Taxus Liberté PES had a tendency toward a decreased incidence of PLIA with a lower incidence of subclinical thrombus than the lesions treated with the Taxus Express PES. Although still speculative, we suggest that the homogenous drug elution and decreased local injury afforded by the Taxus Liberté PES might explain the differences observed between the Taxus Liberté PES and the Taxus Express PES. Farb et al have demonstrated that incomplete healing, consisting of persistent intimal fibrin deposition, intraintimal hemorrhage, and increased intimal and adventitial inflammation, was observed only in rabbit iliac lesions treated with higher-dose PES (42.0 mg and $20.2 \mathrm{mg}$ ). ${ }^{24}$ Considering the local toxicity secondary to paclitaxel overdose, homogenous drug distribution afforded by the new platform might have more important implications for clinical practice. A largescale randomized study is required to test our hypothesis.

\section{Study Limitations}

Several limitations of the present study should be noted. First, it was a non-randomized, retrospective study based on a relatively limited sample size, which raises the possibility of selection bias. Also, the follow-up period was limited to 6 months, a time at which neointimal hyperplasia in response to vessel wall injury from stenting is substantially but not fully complete and no insight can be provided into late events such as very late stent thrombosis. These limitations warrant further studies in additional patients receiving longer follow-up. Second, this study examined PES only, without any comparison to BMS or other DES. Third, because approximately $10 \%$ of enrolled patients did not receive dual antiplatelet therapy at the time of follow-up OCT, the incidence of thrombus formation under dual antiplatelet therapy is still unclear. In the present study, the incidence of subclinical thrombus was similar between patients who were taking only aspirin and those who were on dual antiplatelet therapy. Even on dual antiplatelet therapy, an incidence of thrombus formation of 30-40\% has been reported and several cases of newly formed thrombus were also observed. ${ }^{25,26}$ Therefore, although dual antiplatelet therapy is an important factor to reduce the risk of clinical thrombosis, it may not be effective enough to completely suppress thrombus formation after PES implantation. Fourth, although we demonstrated the comparability of tissue characteristics of target plaque using conventional gray-scale IVUS, it may not be satisfactory for plaque characterization. More detailed assessment using OCT or color IVUS will be required for the evaluation of the impact of stent platform on vessel reaction after stenting. Finally, the clinical relevance of the present findings is still unclear, warranting a larger study with a longer follow-up period.

\section{Disclosures}

Conflict of Interest: None declared.

\section{References}

1. Dotter CT. Transluminally-placed coilspring endarterial tube grafts: Long-term patency in canine popliteal artery. Invest Radiol 1969; 4: 329-332.

2. Rogers C, Edelman ER. Endovascular stent design dictates experimental restenosis and thrombosis. Circulation 1995; 91: 2995-3001.

3. Barth KH, Virmani R, Froelich J, Takeda T, Lossef SV, Newsome J, et al. Paired comparison of vascular wall reactions to Palmaz stents, Strecker tantalum stents, and Wallstents in canine iliac and femoral arteries. Circulation 1996; 93: 2161-2169.

4. Garasic JM, Edelman ER, Squire JC, Seifert P, Williams MS, Rogers C. Stent and artery geometry determine intimal thickening independent of arterial injury. Circulation 2000; 101: 812-818.

5. Briguori C, Sarais C, Pagnotta P, Liistro F, Montorfano M, Chieffo A, et al. In-stent restenosis in small coronary arteries: Impact of strut thickness. J Am Coll Cardiol 2002; 40: 403-409.

6. Kastrati A, Mehilli J, Dirschinger J, Dotzer F, Schuhlen H, Neumann FJ, et al. Intracoronary stenting and angiographic results: Strut thickness effect on restenosis outcome (ISAR-STEREO) trial. Circulation 2001; 103: 2816-2821.

7. Edelman ER, Seifert P, Groothuis A, Morss A, Bornstein D, Rogers C. Gold-coated NIR stents in porcine coronary arteries. Circulation 2001; 103: 429-434.

8. Hwang CW, Wu D, Edelman ER. Physiological transport forces govern drug distribution for stent-based delivery. Circulation 2001; 104: $600-605$

9. Pache J, Kastrati A, Mehilli J, Schuhlen H, Dotzer F, Hausleiter J, et al. Intracoronary stenting and angiographic results: Strut thickness effect on restenosis outcome (ISAR-STEREO-2) trial. J Am Coll Cardiol 2003; 41: 1283-1288.

10. Bezerra HG, Costa MA, Guagliumi G, Rollins AM, Simon DI. Intracoronary optical coherence tomography: A comprehensive review clinical and research applications. JACC Cardiovasc Interv 2009; 2: $1035-1046$

11. Hur SH, Hassan AH, Rekhi R, Ako J, Shimada Y, Nakamura M, et al. Serial intravascular ultrasonic study of outcomes of coronary culprit lesions with plaque rupture following bare metal stent implantation in patients with angina pectoris. Am J Cardiol 2007; 99: 13941398.

12. Takebayashi H, Mintz GS, Carlier SG, Kobayashi Y, Fujii K, Yasuda $\mathrm{T}$, et al. Nonuniform strut distribution correlates with more neointimal hyperplasia after sirolimus-eluting stent implantation. Circulation 2004; 110: 3430-3434.

13. Hasegawa T, Ako J, Ikeno F, Waseda K, Suzuki Y, Honda Y, et al. 
Comparison of nonuniform strut distribution between two drug-eluting stent platforms. J Invasive Cardiol 2007; 19: 244-246.

14. Matsumoto D, Shite J, Shinke T, Otake H, Tanino Y, Ogasawara D, et al. Neointimal coverage of sirolimus-eluting stents at 6-month follow-up: Evaluated by optical coherence tomography. Eur Heart $J$ 2007; 28: 961-967.

15. Otake H, Shite J, Ako J, Shinke T, Tanino Y, Ogasawara D, et al. Local determinants of thrombus formation following sirolimus-eluting stent implantation assessed by optical coherence tomography. JACC Cardiovasc Interv 2009; 2: 459-466.

16. Kume T, Akasaka T, Kawamoto T, Ogasawara Y, Watanabe N, Toyota E, et al. Assessment of coronary arterial thrombus by optical coherence tomography. Am J Cardiol 2006; 97: 1713-1717.

17. Jang IK, Tearney GJ, MacNeill B, Takano M, Moselewski F, Iftima $\mathrm{N}$, et al. In vivo characterization of coronary atherosclerotic plaque by use of optical coherence tomography. Circulation 2005; 111: $1551-1555$.

18. Teramoto T, Ikeno F, Otake H, Lyons JK, van Beusekom HM, Fearon $\mathrm{WF}$, et al. Intriguing peri-strut low-intensity area detected by optical coherence tomography after coronary stent deployment. Circ J 2010; 74: $1257-1259$.

19. Otake H, Shite J, Ikeno F, Shinke T, Teramoto T, Miyoshi N, et al. Evaluation of the peri-strut low intensity area following sirolimusand paclitaxel-eluting stents implantation: Insights from an optical coherence tomography study in humans. Int J Cardiol 2012; 157: $38-42$.

20. Bland JM, Altman DG. Statistical methods for assessing agreement between two methods of clinical measurement. Lancet 1986; 1: 307-310.

21. Shite J. Delayed neointimalization on drug-eluting stents: Speculation from optical coherence tomography. Circ J 2009; 73: 22102211.

22. Nakagawa M, Shite J, Shinke T, Otake H, Okada K, Okita Y, et al. Ability of optical coherence tomography to visualize the entry port of spontaneous coronary artery dissection. Circ J 2011; 75: 25052507.

23. Turco MA, Ormiston JA, Popma JJ, Hall JJ, Mann T, Cannon LA, et al. Reduced risk of restenosis in small vessels and reduced risk of myocardial infarction in long lesions with the new thin-strut TAXUS Liberte stent: 1-year results from the TAXUS ATLAS program. JACC Cardiovasc Interv 2008; 1: 699-709.

24. Jabara R, Geva S, Ribeiro HB, Chen JP, Hou D, Li J, et al. A third generation ultra-thin strut cobalt chromium stent: Histopathological evaluation in porcine coronary arteries. EuroIntervention 2009; 5: $619-626$.

25. Awata M, Kotani J, Uematsu M, Morozumi T, Watanabe T, Onishi T, et al. Serial angioscopic evidence of incomplete neointimal coverage after sirolimus-eluting stent implantation: Comparison with baremetal stents. Circulation 2007; 116: 910-916.

26. Takano M, Yamamoto M, Xie Y, Murakami D, Inami S, Okamatsu $\mathrm{K}$, et al. Serial long-term evaluation of neointimal stent coverage and thrombus after sirolimus-eluting stent implantation by use of coronary angioscopy. Heart 2007; 93: 1533-1536. 